\title{
MUDANÇAS CLIMÁTICAS COMO UM FATOR EXÓGENO QUE AFETA O COMPORTAMENTO DE BUSCA TECNOLÓGICA
}

\author{
Paulo Henrique Assis Feitosa \\ Universidade Estadual de Campinas (Unicamp)
}

\begin{abstract}
Resumo
Este artigo tem como objetivo examinar o processo de mudanças climáticas globais como um fator exógeno que modifica o grau e a direção do comportamento de busca tecnológica das firmas. A análise organiza a literatura principal em três níveis principais, da firma, dado o esgotamento tecnológico e a expansão para além de mercados nacionais, do mercado, diante de condições específicas da sua evolução que afetam o seu funcionamento e a distribuição de incentivos, e do sistema, que reflete os estímulos para realização de atividades inovativas a partir de diferentes condições de oportunidade tecnológica. Procura-se demonstrar, a partir da literatura subjacente, que as transformações no ambiente tecnológico promovidas pelas mudanças climáticas constituem um importante fator exógeno capaz de alterar a forma como as firmas conduzem sua atividade de busca tecnológica. Nesse sentido, a contribuição do artigo consiste em relacionar essas transformações em três níveis principais, de forma a extrapolar os limites setoriais, tecnológicos e geográficos dos estudos existentes.
\end{abstract}

Palavras-chave: Mudanças climáticas; busca tecnológica; exploração e explotação.

\section{CLIMATE CHANGE AS AN EXOGENOUS TRIGGERS AFFECTING THE TECHNOLOGICAL SEARCH BEHAVIOR}

\begin{abstract}
This paper aims to examine the process of global climate change as an exogenous trigger that modifies the level and direction of the firms' technological search behavior. The analysis organizes the main literature on three main levels, the firm, given the technological exhaustion and expansion beyond national markets, the market, given specific conditions of its evolution affecting its operation and the distribution of incentives, and the system, which reflects the stimuli for carrying out innovative activities from different technological opportunity conditions. The paper seeks to demonstrate, from the underlying literature, that the transformations in the technological environment promoted by the climatic changes are an important exogenous factor capable of changing the way in which companies lead their technological search activity. In this sense, the contribution of the article is to relate these transformations to three main levels, in order to extrapolate the sectorial, technological and geographical limits of the existing studies.
\end{abstract}

Keywords: Climate changes; technological search; exploitation and exploitation.

Área ENEI 5: Inovação e mudanças técnica, organizacional e institucional.

Classificações JEL: O31, O33, Q54, Q55. 


\section{Introdução}

A literatura tradicional convencionou que o processo de busca é conduzido majoritariamente de forma local (DOSI, 1982; NELSON; WINTER, 1982; PATEL; PAVITT, 1997), contudo, é possível perceber mudanças na ênfase das interpretações sobre o comportamento da firma ao longo da literatura posterior que foi crescentemente modificadas para um foco maior em como as firmas equilibram a busca local com a busca exploratória (não local).

Essa literatura posterior, ainda que reconheça o fato de que os processos de busca permaneçam predominantemente locais, procura analisar como firmas podem evitar a armadilha da busca local e equilibrar esse modo de busca com a busca exploratória (FLEMING; SORENSON, 2004; LAURSEN, 2012). A mudança de ênfase parte do questionamento da conveniência da busca local em todos os contextos, tanto numa perspectiva teórica quanto empírica, e tende a afirmar que a dependência da busca local pode levar prejuízos às firmas, ou ainda à sua própria extinção.

As desvantagens da busca local podem ser observadas em muitas situações, particularmente naquelas em que o conhecimento necessário para solução dos problemas não coincide com a base de conhecimento das firmas. Nesse caso, a busca local pode ser insuficiente para proporcionar a variedade de conhecimentos necessária para a sua resolução, ou ainda para gerar oportunidades de combinação e recombinação de conhecimentos. A ênfase na busca local tende a gerar comportamentos míopes e vieses cognitivos, ou seja, situações em que soluções superiores, por pertencerem a domínios de conhecimento distantes das firmas, são sistematicamente negligenciadas. O acesso das firmas a uma variedade de recursos é, portanto, um fator crítico e a busca exploratória é motivada pela possibilidade de um alargamento das suas fronteiras tecnológicas que permita que elas encontrem fontes de variedade necessárias à criação de conhecimentos e a novas combinações de tecnologias.

No debate sobre o equilíbrio entre busca local e exploratória, uma questão fundamental é como os fatores exógenos podem levar a modificações no comportamento de busca das firmas. Esse questionamento parte do diagnóstico da existência de lacunas em investigações sobre como esses fatores modificam o nível e a direção do comportamento de busca das firmas. Contudo, qualquer estudo que se proponha a lidar com essas limitações deve considerar o desafio de identificar e analisar modificações no ambiente tecnológico suficientemente abrangentes, e que não sejam específicos de setores industriais, áreas tecnológicas ou localidades geográficas.

Este artigo advoga que os efeitos das mudanças climáticas sobre o comportamento das firmas constituem um contexto oportuno para investigação nesse aspecto. A mitigação das emissões de gases de efeito estufa (GEE) é um desafio global e exige busca local e exploratória para a reestruturação de ativos tecnológicos constituídos por firmas ao longo de décadas. Ao mesmo tempo, as tecnologias de mitigação tendem a ser abrangentes, horizontais e pervasivas, e desta forma, garantem uma importante oportunidade de investigação de mudanças no grau e direção do comportamento de busca tecnológica das firmas.

Diante deste contexto, o artigo tem como objetivo examinar o processo de mudanças climáticas globais como um fator exógeno que modifica o grau e a direção do comportamento de busca tecnológica das firmas. Desta forma, propõe-se a análise esse fator exógeno em três níveis principais: i) da firma, em que se destacam circunstâncias que podem encorajar firmas a investir em processos de busca, dentre as quais se destacam o esgotamento tecnológico e a expansão para além de mercados nacionais; ii) do mercado, que é caracterizado por condições específicas da sua formação e evolução que afetam o seu funcionamento e a distribuição de incentivos, como a existência de falhas e externalidades; iii) o nível do sistema, que a partir de diferentes condições de oportunidade tecnológica, reflete os incentivos para a realização de atividades inovativas. 
O artigo está organizado em cinco seções, além desta introdução. A segunda seção discute os aspectos teóricos e conceituais das rotinas organizacionais e os processos de busca. A terceira apresenta o debate sobre busca exploratória e capacidade de absorção. A discussão sobre os fatores exógenos que afetam o processo de busca é exposta na quarta seção e a última seção apresenta as considerações finais.

\section{Rotinas organizacionais e processos de busca}

A literatura tradicional trata as rotinas organizacionais como uma regra importante de comportamento das firmas, que podem ser definidas como um conjunto padronizado de regras e heurísticas incorporadas, ou ainda "pedaços" de atividades que possuem um caráter repetitivo (DOSI; NELSON; WINTER, 2001). Em algum momento, as firmas "[...] have built into them a set of ways of doing things and ways of determining what to do" (NELSON; WINTER, 1982, p. 400). Como rotinas não são imutáveis, as firmas realizam atividades de busca sempre que precisam avaliar as rotinas atuais que levem a sua modificação, mudanças ou substituição (NELSON; WINTER, 1982, p. 400). Contudo, os autores também reconhecem que existem limites à flexibilidade desse comportamento, e que um ambiente em mudanças pode forçar as firmas a arriscar sua própria sobrevivência em tentativas de modificar suas rotinas.

As atividades de busca que avaliam as rotinas mantidas pela firma podem ser conduzidas de duas formas principais. Enquanto a busca local tende a relacionar o conhecimento que está no entorno da base de conhecimento atual da organização, a busca não local "envolve um esforço consciente para se afastar das rotinas organizacionais e das bases de conhecimento atuais" (KATILA; AHUJA, 2002, p. 1184). Tradicionalmente, a literatura enfatiza que esses processos de busca são realizados de forma local (DOSI, 1982; NELSON; WINTER, 1982; PATEL; PAVITT, 1997). Deste modo, enfatiza a tendência das firmas em se concentrar em campos tecnológicos que compartilham a mesma base de conhecimento e semelhança na resolução de problemas (NELSON; WINTER, 1982). Assim, as firmas precisam obter o máximo de vantagem do conjunto de conhecimento que possuem, o que requer concentração, ou, no mínimo, coerência, mais do que a distribuição aleatória dos seus esforços (NELSON, 1991, p. 68).

O processo de busca é majoritariamente local, pois tende a ocorrer ao longo de trajetórias estabelecidas pela experiência passada, rotinas e heurísticas (WINTER, 1984, p. 292). Como as mudanças tecnológicas radicais são incomuns, com frequência o progresso tecnológico emerge ao longo de uma trajetória estabelecida pelo paradigma tecnológico vigente. Esses paradigmas funcionam como um modelo e um padrão de solução de problemas baseados em princípios selecionados, e incorporam fortes prescrições para as direções de mudança técnica que devem ser perseguidas ou ignoradas (DOSI, 1982, p. 152).

Uma das principais justificativas para a predominância da busca local é o custo para se dominar conhecimentos especializados no mundo contemporâneo, principalmente em grandes firmas, que detêm conhecimentos em numerosos campos tecnológicos (CANTWELL; FAI, 1999; PAVITT, 1998, p. 441). Outra razão é a dificuldade envolvida na transformação de tecnologias em produtos rentáveis pelo fato de abrangerem muitas tecnologias distintas ${ }^{1}$ (PATEL; PAVITT, 1997). Como resultado, as firmas utilizam a busca local em detrimento da exploratória (não-local) por ser uma estratégia mais eficiente e vantajosa, além de apresentar custos relativamente mais baixos (LAURSEN, 2012).

Essa abordagem pressupõe que grande parte do conhecimento mantido pelas firmas é tácito (NELSON; WINTER, 1982), característica que torna mais difícil, e muitas vezes impossível, a tarefa de transferir o conhecimento incorporado em indivíduos e firmas em contextos distantes. Como a assimilação de novos

\footnotetext{
${ }^{1}$ Um exemplo é a produção de veículos motorizados, que exige o domínio de tecnologias de transporte, mas também químicas, elétricas e eletrônicas (PATEL; PAVITT, 1997, p. 145).
} 
conhecimentos pela firma ocorre por meio de um processo de aprendizagem gradual, a atividade de busca tende a se restringir a áreas próximas, nas quais ela já possui domínio (ROSENKOPF; ALMEIDA, 2003, p. 752).

De modo geral, as firmas tendem a aprofundar a sua compreensão de elementos locais que potencialmente poderiam ser combinados, tornando-as mais capazes de inventar e, com maior confiabilidade, evitar as combinações que não funcionaram no passado (FLEMING; SORENSON, 2004, p. 911). Desta forma, existem vantagens evidentes para as firmas na realização de buscas locais em suas atividades de resolução de problemas, incluindo aqueles relacionados à inovação.

De acordo com Nelson e Winter (1982), essas firmas são muito melhor em "[...]tasks of self-maintenance in a constant environment than they are at major change, and much better at changing in the direction of 'more of the same' than they are at any other kind of change" (NELSON; WINTER, 1982, p. 8-9). Da mesma forma, Dosi (1988) sintetiza as razões para busca local ao afirmar:

The search process of industrial firms to improve their technology is not likely to be one where they survey the whole stock of technological knowledge before making their technical choices. Given its highly differentiated nature, firms will instead seek to improve and to diversify their technology by searching in zones that enable them to use and to build upon their existing technological base (DOSI, 1988, p. 225).

Essa literatura mostra um comportamento dominante da busca local que se mantém em diferentes conjunturas. Logo, em contextos marcados pela grande complexidade na resolução de problemas, a tendência à busca local pode ser justificada por duas razões principais. A primeira é a existência de um comportamento racional limitado (SIMON, 1997), que faz com que os agentes sejam incapazes de considerar todas as opções possíveis para a solução de seus problemas tecnológicos e inábeis de avaliar com precisão as perspectivas futuras de desempenho das opções potenciais. Tais fatores fazem com que a avaliação de alternativas tenda a ser imperfeita (KNUDSEN; LEVINTHAL, 2007, p. 51-52). A segunda razão é que a base de conhecimento acumulado facilita a aprendizagem relacionada à solução de problemas complexos. Em outras palavras, o aprendizado tende a ser mais fácil se for restrito a vizinhanças familiares e próximas, e isso é observado pelo fato de as inovações incrementais constituírem a maior parte do avanço tecnológico.

A prevalência da busca local também foi corroborada por evidências empíricas. Patel e Pavitt (1997), num estudo a partir da atividade de patenteamento de 440 firmas, revelaram que mais de $90 \%$ delas possuem perfil tecnológico bastante similar quando comparados aos períodos 1969-1974 e 1985-1990. Ao classificar a atividade tecnológica das firmas em cinco grandes áreas, os autores estimaram que o grau de correlações entre os períodos variou entre 0,55 e 0,91 .

Os resultados de Patel e Pavitt (1997), em contraste com evidências sobre o processo de busca encontradas posteriormente, induziram a um debate sobre a existência de um possível paradoxo da variedade (LAURSEN, 2012), que questiona se o desempenho inovador das firmas é determinado por sua capacidade de transformar tecnologias em produtos, ou pela sua capacidade de desenvolver tecnologias por meio da busca por variedade (ou ambos).

Patel e Pavitt (1997), sugerem que a variedade é uma característica mais necessária para mudanças técnicas revolucionárias e menos para mudanças normais. Os autores também afirmam que nesse debate é preciso diferenciar tecnologias e produtos. Desta forma, como as tecnologias podem ser combinadas numa ampla configuração de produtos, é possível que, em um determinado setor econômico, a variedade de produtos coexista com homogeneidade de tecnologias. Entretanto, esse argumento contrasta fortemente com a abordagem evolucionista tradicional, que afirma que os produtos das firmas são diferentes porque suas 
tecnologias são heterogêneas. Os autores argumentam que, em vez disso, as firmas dentro do mesmo setor industrial possuem mais ou menos as mesmas tecnologias, porém diferem internamente em relação à forma como podem transformar essas tecnologias em produtos de sucesso no mercado.

Para Laursen (2012), essa perspectiva guarda um importante poder explicativo, mas não é a única possível. Ainda que os processos de busca tecnológica sejam marcados por fortes imperativos, os limites impostos por esses imperativos podem ser amplos o suficiente para explicar uma proporção significativa da variação do desempenho inovador no nível da firma. O autor procura incorporar duas questões complementares nesse debate, a primeira é o aspecto intertemporal do processo de busca tecnológica, que induz firmas líderes a realizar busca exploratória e em seguida introduzir novas tecnologias no mercado, ganhando uma vantagem temporária em relação aos concorrentes. A segunda é que as firmas concorrentes dependem não apenas da sua capacidade interna de gerar inovações, mas principalmente da apropriação de tecnologias geradas por seus concorrentes.

A exploração do espaço tecnológico em campos emergentes é fundamental para que as firmas absorvam oportunidades futuras de negócios e também para que reduzam o risco de se tornarem obsoletas do ponto de vista tecnológico. Patel e Pavitt (1997) afirmam que, pelo menos nos estágios iniciais, um campo emergente "will be marginal in the total technological portfolio of the firm, but this will change as a function of the richness of the stream of potential opportunities that are identified" (PATEL; PAVITT, 1997, p. 148). Entretanto, é preciso reconhecer que é improvável que as atividades de busca exploratória sejam a estratégia dominante de firmas bem-sucedidas, devido ao seu alto custo se comparada com a busca local (LAURSEN, 2012).

Para além da aparente contradição entre as evidências de Patel e Pavitt (1997) e a literatura sistematizada por Laursen (2012), existem convergências importantes. Alguns estudos revelam que as firmas que mantém altos níveis de atividades exploratórias são penalizadas com menor nível de inovação em produto, se comparadas com as demais firmas (KATILA; AHUJA, 2002; LAURSEN; SALTER, 2006). Em outras palavras, existe um relativo consenso entre os estudiosos de que a busca por variedade é fortemente limitada e que o conjunto de conhecimentos mantidos pela firma determina, dada a existência de limites cognitivos, sua capacidade de busca e seu potencial produtivo (DOSI, 1988; LAURSEN, 2012; LEVINTHAL; MARCH, 1993; MARCH, 1991; PATEL; PAVITT, 1997).

\section{Busca exploratória e capacidade de absorção}

Se por um lado a literatura tradicional enfatizou o caráter local dos processos de busca (DOSI, 1982; NELSON; WINTER, 1982; PATEL; PAVITT, 1997), é possível perceber mudanças na ênfase das interpretações sobre o comportamento da firma ao longo da literatura subsequente. Laursen (2012), numa revisão panorâmica das principais contribuições empíricas sobre o tema, identificou que a ênfase da literatura tradicional nos processos de busca local foi paulatinamente modificada para um foco maior em como as firmas equilibram a busca local com a busca exploratória (não local).

Essas contribuições posteriores, ainda que pressuponham que os processos de busca permaneçam predominantemente locais, procuram analisar como firmas podem evitar a armadilha da busca local e equilibrar essa busca com a busca exploratória (FLEMING; SORENSON, 2004; LAURSEN, 2012). Tal mudança de ênfase parte do questionamento de estudiosos sobre a inevitabilidade da busca local em todos os contextos, tanto numa perspectiva teórica quanto empírica. Da mesma forma, é cada vez mais reconhecido que a dependência da busca local pode ser prejudicial, ou mesmo letal às firmas (LAURSEN, 2012; LEVINTHAL; MARCH, 1993; TRIPSAS; GAVETTI, 2000). 
A desvantagem da busca local é percebida em muitas situações, especialmente quando o conhecimento necessário para resolver um novo problema pode não coincidir com a base de conhecimento das firmas. Nesse caso, a busca local pode ser insuficiente para proporcionar a variedade de conhecimentos necessária para a resolução de problemas, ou mesmo gerar oportunidades bastantes de combinação e recombinação de conhecimentos (ROSENKOPF; NERKAR, 2001; ROTHAERMEL; ALEXANDRE, 2009). Como sugerido pela literatura, a ênfase na busca local pode levar ao comportamento míope e a vieses cognitivos (LEVINTHAL; MARCH, 1993; MARCH, 1991), ou seja, situações em que soluções superiores, por pertencerem a domínios de conhecimento distantes das firmas, são sistematicamente negligenciadas ${ }^{2}$. O acesso das firmas a uma variedade de recursos é, portanto, um fator crítico e a busca exploratória é motivada pela possibilidade de um alargamento das suas fronteiras tecnológicas que permita que elas encontrem fontes de variedade necessárias à criação de conhecimentos e a novas combinações de tecnologias.

Essa abordagem foi originalmente inspirada em March (1991), que propôs uma distinção entre duas formas de comportamento organizacional: de um lado, a exploração (exploration), que compromete indivíduos e organizações na busca, experimentação e variação; do outro, a explotação (exploitation), que aumenta a produtividade e eficiência por meio da escolha, execução e redução da variedade. ${ }^{3}$ Ambos os comportamentos são essenciais para os processos de aprendizagem e crescimento organizacional, mas implicam em contradições inerentes a mudanças incrementais e revolucionárias que precisam ser gerenciadas (TUSHMAN; O'REILLY, 1996). Diversos estudos ampliaram esse arcabouço analítico a partir da sua aplicação em uma gama de fenômenos, que por um lado abrangem várias manifestações de especialização e experiência, e por outro a diversidade e experimentação (LAVIE; STETTNER; TUSHMAN, 2010).

A proposta de March (1991), dada a sua amplitude, foi utilizada em vários contextos, como o desenvolvimento tecnológico, inovação de produto e alianças estratégias, bem como em vários níveis de análise, tais como indivíduos, grupos, organizações e indústrias ${ }^{4}$. Levinthal e March (1993, p. 105) limitaram o âmbito destas atividades para o domínio do conhecimento afirmando que a exploração envolve a busca de novos conhecimentos, enquanto a explotação envolve o uso e desenvolvimento de coisas já conhecidas. Diante da ampla aceitação e influência dessa abordagem, estudos têm se empenhado em distinguir o desenvolvimento e utilização do conhecimento, relacionando os conceitos de exploração e explotação à escala e escopo do conhecimento criado ou adquirido em relação à base de conhecimento mantido pela firma.

Mesmo se aplicadas definições mais restritas, permanecem importantes divergências entre estudiosos. Para alguns, a explotação pode envolver o desenvolvimento de conhecimento (HE; WONG, 2004) e, para outros, a explotação se resume ao desenvolvimento do conhecimento existente (VERMEULEN; BARKEMA, 2001). A distinção entre exploração e explotação torna-se uma tarefa não trivial dada a multidimensionalidade da variável conhecimento organizacional (LAVIE; STETTNER; TUSHMAN, 2010). Como a geração de novos conhecimentos depende, de certa maneira, da base de conhecimento mantida pela firma (COHEN; LEVINTHAL, 1990), é preciso reconhecer que os estudos empíricos com essa abordagem sempre enfrentarão dificuldades em distinguir explotação e exploração.

\footnotetext{
${ }^{2}$ Um exemplo clássico é a síndrome do não inventado aqui (not invented here), em que firmas evitam soluções de terceiros apenas por causa de sua origem externa (KATZ; ALLEN, 1982).

3 Como definido por March (1991, p. 71), a explotação envolve "refinamento, escolha, produção, eficiência, seleção, implementação e execução", enquanto a exploração é composta por "pesquisa, variação, riscos, experimentação, flexibilidade, descoberta e inovação".

${ }^{4}$ Para uma síntese dessas interpretações, vide Lavie, Stettner e Tushman (2010).
} 
De acordo com Lavie, Stettner e Tushman (2010, p. 114), a explotação está sempre associada à base de conhecimento mantida pela firma. Deste modo, enquanto essa firma persistir em uma trajetória tecnológica existente e utilizar suas competências internas para tal, suas operações estarão voltadas para a explotação. Por outro lado, a exploração requer uma mudança na base atual de conhecimentos e suas competências. Essas mudanças podem estar relacionadas a novas habilidades técnicas, ao conhecimento do mercado ou ainda às relações externas.

É importante reconhecer a existência de um ciclo natural exploração-explotação e que a transitividade entre ambas ao longo do tempo é mais bem descrita com um continuum do que apenas um modelo de escolha discreta. Entretanto, como a capacidade que a firma possui de gerar e desenvolver novos conhecimentos depende de sua base de conhecimento, a exploração é substituída pela explotação com a subsequente aplicação do conhecimento (COHEN; LEVINTHAL, 1990; LAVIE; STETTNER; TUSHMAN, 2010).

Um exemplo é uma firma que experimenta uma nova tecnologia e legitima a exploração. Na medida em que repete esses experimentos e a aplicação de novos conhecimentos adquiridos, ela desenvolve rotinas de explotação e se torna mais familiarizada com esse conhecimento. Portanto, existe um circuito no qual a exploração tende a evoluir para a explotação, especialmente em determinados contextos, como é o caso do processo de inovação (BRUNNER et al., 2009; LAVIE; STETTNER; TUSHMAN, 2010).

Os benefícios para firmas que alcançam e mantém um balanceamento entre suas estratégias de explotação e exploração tem sido o objeto de estudos sobre o tema, ainda que pouco consenso tenha sido construído sobre as formas pelas quais as firmas alcançam esse equilíbrio (ADLER et al., 2009; GUPTA et al., 2006, p. 697). Conceitualmente, a capacidade de englobar níveis relativamente altos de exploração e exploração é percebida em organizações ambidestras (TUSHMAN; O'REILLY, 1996), entretanto, as tensões e conflitos permanentes entre essas atividades podem exigir a sua separação no contexto organizacional.

Pode-se identificar três modos fundamentais pelos quais as firmas lidam com os conflitos permanentes entre exploração e explotação. O primeiro modo está marcado pela ambidestria contextual, em que a exploração e a explotação ocorrem simultaneamente, mas dentro de unidades organizacionais distintas. No segundo modo, há a separação temporal, em que ambas as atividades ocorrem na mesma organização, mas alternadas em diferentes momentos. Por fim, pode haver uma separação de domínio, no qual as firmas se especializam em exploração ou explotação em campos organizacionais específicos e equilibram suas atividades entre diferentes domínios (LAVIE; STETTNER; TUSHMAN, 2010).

Existe um relativo consenso entre estudiosos de que o processo de busca por variedade é fortemente limitado por sua capacidade de absorção, ou seja, pelo alcance da sua aptidão em reconhecer o valor de novas informações, assimilá-las e aplicá-las para fins comerciais. Desde a contribuição seminal de Cohen e Levinthal (1990), a literatura tem aprimorado e estendido a noção de capacidade de absorção e suas repercussões sobre as firmas. Zahra e George (2002) propõem uma divisão da capacidade de absorção em duas principais, a potencial, que se refere à capacidade das firmas em adquirir e assimilar conhecimento gerado externamente, e a realizada, que está associada à forma como transformam e exploram esse conhecimento para fins comerciais. Deste modo, a inclinação das firmas em adquirir e assimilar o conhecimento gerado externamente está limitado por sua capacidade de absorção potencial.

A partir da abordagem proposta por Zahra e George (2002), o estudo de Jansen, van den Bosch e Volberda (2005) analisa em que medida os mecanismos organizacionais como coordenação e socialização explicam o fato de algumas firmas serem capazes de adquirir e assimilar novos conhecimentos externos, mas não de transformá-los e explorá-los com sucesso. Os resultados revelam como unidades organizacionais diferem em sua capacidade de criar valor a partir de sua capacidade de absorção. Já o trabalho de Fosfuri e Tribó (2008) mostra que, durante períodos de mudanças na estratégia, as firmas se esforçam para acumular 
capacidade de absorção potencial e que os fluxos de conhecimento internos ajudam a reduzir a distância entre a capacidade de absorção potencial e realizada.

Os limites à capacidade de absorção potencial são explicados pela literatura pela dificuldade em equilibrar os processos de busca local e não local, que resulta em buscas disfuncionais, ou seja, estratégias muito extensas ou excessivamente estreitas, ou ainda a ênfase em determinada forma de busca em detrimento de outras (HE; WONG, 2004; KATILA; AHUJA, 2002; LAURSEN; SALTER, 2006; ROSENKOPF; ALMEIDA, 2003).

Esses desequilíbrios têm causas diversas, como as falhas na projeção da estrutura organizacional ideal para obter o melhor trade-off entre explotação e exploração (LAURSEN, 2012), e a restrição imposta pela escassez de recursos diversos, a destacar o tempo. Como foi descrito por Levinthal e March (1993), o processo de busca é frequentemente conduzido sob pressões extremas de tempo, que geram falhas na determinação de sua extensão, especialmente em contextos em que o ambiente tecnológico muda rapidamente. Como consequência, uma proporção considerável das firmas pode simplesmente ser incapaz de prever o esforço de busca que conduza a um nível ótimo de diversidade, limitando o seu potencial de escolha estratégica.

Essas falhas resultam de capacidades cognitivas limitadas dos agentes e da necessidade de concentrar sua atenção num conjunto restrito de alternativas potenciais, ao invés de pesquisar o ambiente como um todo ou seguir simultaneamente muitos caminhos diferentes (GAVETTI; LEVINTHAL, 2000). Fortemente relacionado a esse problema está o viés cognitivo contra fontes de conhecimento externas, que resultam em processo de busca míope (LEVINTHAL; MARCH, 1993; MARCH, 1991). Nesse caso, o processo de busca é dificultado pela falta de recursos de todas as formas, incluindo a inexistência de uma equipe interna apta a explorar todas as combinações possíveis.

Esse argumento tem influenciado parte importante da literatura e estimulado estudos empíricos diversos. Entretanto, o estudo de Lane, Koka e Pathak (2006), numa revisão crítica desses estudos que utilizam o conceito de capacidade de absorção, concluem que grande parte dos autores utiliza de forma indevida a noção originalmente introduzida por Cohen e Levinthal (1989). Como resultado, o uso indiscriminado do conceito de capacidade de absorção, no âmbito de pesquisas sobre aprendizado organizacional, alianças estratégicas e visão baseada em recursos, tem levado à sua reificação e ao sufoco das investigações na área.

\section{Mudanças climáticas como fator exógeno que afeta o processo de busca}

É possível dividir em três níveis principais os fatores exógenos relacionados às mudanças climáticas que afetam o grau e a direção dos processos de busca tecnológica. O primeiro é o nível da firma, que é marcado por circunstâncias que podem encorajar firmas a investir em processos de busca, dentre as quais se destacam o esgotamento tecnológico, a expansão para além de mercados nacionais (AHUJA; KATILA, 2004) e pressões sociais (KESIDOU; DEMIREL, 2012). O segundo é o nível de mercado, caracterizado por condições específicas da sua formação e evolução que afetam o seu funcionamento e a distribuição de incentivos, como a existência de falhas e externalidades. O terceiro é o nível do sistema, que, a partir de diferentes condições de oportunidade tecnológica, reflete os incentivos para realização de atividades inovativas (MALERBA; ORSENIGO, 1993). Nesse nível, destacam-se fatores institucionais diversos e as políticas exercem um papel central na condução dos processos de busca ${ }^{5}$.

\footnotetext{
${ }^{5}$ Laursen (2012) sugere ainda que o nível individual pode afetar os processos de busca a partir dos diferentes comportamentos e métodos de pesquisa de cientistas e engenheiros.
} 


\subsection{Fatores no nível da firma}

No que se refere ao nível da firma, alguns fatores externos podem ser elencados como indutores dos processos de busca tecnológica. De acordo com Ahuja e Katila (2004), determinados contextos estimulam firmas a criar caminhos inovativos únicos, que afetam o grau e a direção dos processos de busca tecnológica. Em seu estudo sobre empresas do setor químico com sede nos Estados Unidos, Ahuja e Katila (2004) denominam esses contextos situações idiossincráticas, como o esgotamento tecnológico e a expansão para além de mercados nacionais.

O processo inovativo é um processo de acoplamento e de correspondência, no qual a interação é um elemento crítico (FREEMAN; SOETE, 1997, p. 200). Com frequência, esse processo ocorre através da combinação e recombinação de elementos existentes em novos artefatos. Entretanto, a menos que ao longo do tempo os elementos disponíveis para a recombinação sejam aumentados de alguma forma, o ritmo da inovação deve diminuir à medida em que o espaço disponível para essa recombinação se esgota (FLEMING, 2001). Para lidar com esse cenário, as firmas que já realizam investimentos em áreas onde a tecnologia é bem explorada são induzidas a buscar o acesso a novas fontes de conhecimento (AHUJA; KATILA, 2004).

Outra situação idiossincrática é a ocorrência de uma expansão para além de mercados nacionais. As firmas que operam em vários mercados nacionais estão sujeitas a lidar com as circunstâncias específicas de cada mercado. Nesses casos, as variações nas necessidades dos usuários, nos processos de fabricação ou na disponibilidade de insumos podem exigir a adaptação da tecnologia a contextos locais ou mesmo o desenvolvimento de tecnologias completamente novas (AHUJA; KATILA, 2004). Essa expansão para novos mercados geográficos também pode apresentar diversas oportunidades. A presença em novos mercados aumenta o potencial de busca da firma por meio da participação em redes internas e externas de pesquisa para a criação de conhecimento ou inovação (CANTWELL, 2013).

No caso da busca em áreas com potencial de mitigação das mudanças climáticas, a expansão para novos mercados é marcada por níveis mais elevados de atividade tecnológica internacional se comparada à atividade nas demais tecnologias (DECHEZLEPRÊTRE et al., 2011). Nesse contexto, a expansão dos mercados para a tecnologia tem exercido um papel crucial na estratégia tecnológica das firmas, como meio para viabilizar a busca no ambiente tecnológico internacional. $O$ surgimento e consolidação desses mercados permitiu uma nova janela de oportunidade para as firmas que são mais abertas para o exterior e que estão permanentemente envolvidas em atividade de pesquisa (ARORA; FOSFURI; GAMBARDELLA, 2004). Diante do fato de as firmas recorrerem à busca exploratória de conhecimento para alimentar sua capacidade de inovação, a necessidade de explorar a quantidade crescente de fontes externas de conhecimento afeta o grau e a direção desse processo.

Outros fatores que interferem no processo de busca nessa área são as pressões sociais e as exigências do mercado. O estudo de Kesidou e Demirel (2012, p. 868) mostra que essas pressões e exigências de fato induzem firmas a realizarem investimentos, porém esse esforço é pequeno e insuficiente para incentivar a mobilização de grandes quantidades de recursos para inovação ambiental. Outro resultado desse estudo é que a responsabilidade social corporativa e as necessidades dos clientes para produtos ecológicos não afetam o nível de investimento nessas inovações.

A consciência ambiental dos consumidores e das empresas também é uma variável importante, em se tratando de produtos e serviços verdes (environmentally friendly). Popp, Hafner e Johnstone (2011), num estudo sobre a indústria de papel e celulose nas décadas de 1980 e 1990, mostram que a pressão dos consumidores somada à regulação foram fundamentais para o desenvolvimento de difusão de tecnologias livres de cloro (Chlorine-Free), que possui diversos efeitos nocivos à saúde humana. Para os autores, 
enquanto estudos precedentes enfatizaram a importância fundamental da regulação para induzir essas inovações, esse caso revela que muitas inovações ocorreram antes da regulação ser implementada.

\subsection{Fatores no nível dos mercados}

Muitos dos impactos ambientais das atividades econômicas, como a emissão de gases poluentes, são casos típicos de externalidades, ou seja, são o efeito de uma atividade cujas consequências são sofridas por uma ou várias partes que não sejam aquelas que controlam a atividade que produziu a externalidade ${ }^{6}$. No funcionamento de um sistema econômico, enquanto o custo para o uso de recursos produtivos, como matéria-prima e trabalho, é internalizado pelas firmas ao pagar por seu uso, não existem incentivos econômicos para minimizar os custos externos das emissões e das externalidades em geral ${ }^{7}$.

De acordo com a literatura econômica clássica, diante da presença de falhas de mercado, alguns dos pressupostos de teoremas do bem-estar não podem ser invocados para produzir um resultado ótimo de Pareto. Essas situações exigem intervenções com o objetivo de atenuar estes desequilíbrios, aumentando o incentivo para as empresas minimizarem as externalidades pela imposição de um limite para o nível das emissões e a internalização dos custos ambientais.

Numa perspectiva de curto prazo, essa literatura demonstra que a eficiência destas políticas exige a comparação do custo marginal da redução das emissões com o benefício marginal de uma atmosfera mais limpa. Em termos estáticos, enquanto as emissões de gases mais danosos devem ser fortemente restringidas pelos altos custos marginais impostos à sociedade, a emissão de gases cuja eliminação é mais onerosa deve ser tolerada, diante do alto custo marginal em reduzi-las (JAFFE; NEWELL; STAVINS, 2005).

Por outro lado, a inclusão da mudança técnica nesta equação altera o trade-off entre custo marginal do controle das emissões e os benefícios sociais marginais, já que diversas tecnologias, como, por exemplo, os métodos de controle de emissões e produção limpa, tendem a reduzir o custo marginal para se alcançar uma determinada unidade de redução das emissões. Como resultado, as introduções de mudanças técnicas possibilitam que um determinado nível de qualidade ambiental seja alcançado a um menor custo total para a sociedade.

O alcance de níveis de mudança técnica requeridos não é trivial e a ação de livres forças de mercado resulta num nível de investimento privado em tecnologias ambientais inferior ao ideal socialmente desejado. A principal justificativa é o surgimento de falhas de mercado no processo de invenção, inovação e adoção (ou difusão) tecnológica. A conjunção dessa falha com as externalidades ambientais negativas das emissões de poluentes resulta no que a literatura denomina como o problema da dupla externalidade (RENNINGS, 2000).

A identificação dessas falhas de mercado pela literatura parte do reconhecimento de que a atividade inovativa não acontece no vácuo, mas constitui um processo mais complexo do que se costuma considerar (JAFFE; NEWELL; STAVINS, 2005). A fase de invenção exige pesquisa científica e de engenharia para transformar ideias em produtos e em processos comerciais. Já na fase de adoção, exige-se o esforço de aprendizado sobre a nova tecnologia, aquisição de novos equipamentos e adaptação das tecnologias a circunstâncias particulares. Como resultado dessa abordagem, torna-se possível identificar falhas de

\footnotetext{
${ }^{6}$ É uma típica manifestação de falhas de mercado, onde o bem-estar do consumidor ou a possibilidade de produção da firma são diretamente afetados pela ação de outro agente na economia (MAS-COLELL; WHINSTON; GREEN, 1995, p. 352).

${ }^{7}$ Existe uma longa tradição no estudo destas questões, a destacar o princípio do poluidor pagador de Pigou (1920) e Baumol \& Oates (1971 e 1998).
} 
mercado em cada uma das fases do processo, que podem ser classificadas em três grupos principais: as externalidades de conhecimento, as externalidades de adoção e de difusão, e a informação incompleta.

No primeiro grupo, as externalidades de conhecimento resultam da dificuldade que os agentes econômicos enfrentam na captura dos benefícios da inovação. Ainda que alguns tenham mais sucesso em obter retornos do seu investimento, ele é sempre uma fração dos benefícios gerais desta inovação para a sociedade como um todo. Em outras palavras, as inovações geram de spillovers de conhecimento para outras firmas e de spillovers de valor ou excedente do consumidor para os usuários da nova tecnologia ${ }^{8}$.

Essa externalidade é resultado da natureza do conhecimento, que faz com que as firmas não consigam evitar que outros também se beneficiem dos seus novos conhecimentos e com que não capturem para si todos os benefícios da inovação. Desta forma, determinada firma, ao produzir uma externalidade com a emissão de GEE, obtém os benefícios econômicos da emissão e impõe os custos das emissões para a sociedade. No caso da tecnologia de mitigação das mudanças climáticas, o problema é inverso, pois a firma que investe ou implementa uma inovação cria benefícios para outros enquanto incorre em todos os custos.

No segundo grupo de falhas de mercado, as externalidades de adoção e difusão tecnológica advêm do fato de o custo ou valor de uma nova tecnologia para um usuário também depender do número de outros usuários que adotarão essa tecnologia. Apenas mais recentemente essa externalidade foi incorporada pela literatura convencional como uma falha de mercado (BERNDT; PINDYCK; AZOULAY, 2000; JAFFE; NEWELL; STAVINS, 2005). O benefício associado com a escala de adoção de uma tecnologia é conhecido como retornos crescentes dinâmicos e são gerados por processos de learning-by-using, learning-by-doing ou externalidades de rede. A adoção e a difusão de uma inovação tende a ser um processo tipicamente gradual e exerce um papel central na determinação de seu sucesso, pois exige que a nova tecnologia seja adaptada a diferentes circunstâncias e prove sua superioridade entre as demais opções existentes.

O processo de learning-by-using afeta a demanda pela tecnologia, pois a sua adoção por um usuário gera uma externalidade positiva para os demais devido à geração de informações sobre a existência, as características e o sucesso da nova tecnologia. Do lado da oferta, o learning-by-doing faz com que fabricantes ganhem experiência com a produção, e se os spillovers de conhecimento beneficiam outros concorrentes sem uma devida compensação, pode representar uma externalidade de adoção adicional. Já as externalidades de rede surgem quando uma determinada tecnologia se torna mais valiosa para um usuário individual a partir do momento em que outros usuários adotam um produto com a mesma tecnologia. Essa externalidade é crítica para a compreensão do sistema tecnológico existente, por auxiliar na previsão de desenvolvimentos tecnológicos ou políticos.

No terceiro grupo de falhas, a informação incompleta é o resultado de assimetria de informações existentes entre os agentes envolvidos em uma transação econômica. Os retornos do investimento em inovação para mitigação das mudanças climáticas envolvem níveis particularmente elevados. Ainda que qualquer investimento numa nova tecnologia envolva um determinado grau de incerteza, existe um grau de incerteza ainda maior sobre os futuros impactos do processo de mudanças climáticas, a magnitude da resposta política e por consequência dos prováveis retornos do investimento em P\&D. Essas questões garantem contornos ainda mais complexos ao problema.

\footnotetext{
${ }^{8}$ Estimativas apontam que a taxa de retorno social média das inovações pode ser de 30\% ou mais (JONES, 2002, p. 220) e as taxas de retorno social dos gastos em P\&D, pelo menos, duas vezes as taxas de retorno privado (JONES; WILLIAMS, 1998, p. 1119).
} 
De modo geral, a informação sobre as perspectivas de sucesso das pesquisas é assimétrica, pois existem dificuldades de antecipação dos resultados da pesquisa básica e alta incerteza sobre efetividade de suas futuras aplicações. Nessas condições, a mudança técnica é operada em ambientes de alto grau de risco já que suas características funcionais variam ao longo do tempo e estão sujeitas a um conjunto complexo de variáveis econômicas e sociais (ALIC; MOWERY; RUBIN, 2003; JAFFE; NEWELL; STAVINS, 2005).

\subsection{Fatores no nível do sistema}

No nível do sistema, os fatores que afetam os processos de busca estão relacionados às oportunidades tecnológicas, ou seja, a variações exógenas no custo e na dificuldade de inovação em diferentes domínios tecnológicos (JAFFE, 1986). Essas variações se devem a características intrínsecas da tecnologia, ou ao estágio de evolução do conhecimento científico num determinado período. Portanto, o nível de oportunidade tecnológica pode mudar ao longo do tempo, mas requer uma quantidade de mudanças significativas para se manifestar.

O conjunto de possibilidades de avanço tecnológico pode ser medido pelo retorno dos gastos em P\&D diante de fatores como as condições de demanda, o estágio de desenvolvimento tecnológico e o regime de apropriabilidade (MALERBA; ORSENIGO, 1993). A existência de elevados níveis de oportunidade tecnológica representa um forte incentivo para condução de processos de busca, ainda que esses incentivos sejam sistematicamente diferentes entre setores e tecnologias. Os níveis elevados de oportunidade permitem uma maior chance para que as firmas combinem e recombinem elementos do conhecimento através da busca tecnológica.

$\mathrm{Na}$ medida em que os recursos são aplicados à $\mathrm{P} \& \mathrm{D}$ e os projetos são concluídos, o conjunto de oportunidades pode se esgotar e as possibilidades de combinação se tornam mais limitadas. Entretanto, como as condições de oportunidade são dinâmicas por natureza, esse conjunto pode ser reabastecido por novas fontes. Klevorick et al. (1995, p. 189), afirmam que as fontes de oportunidade tecnológica se devem: i) aos avanços na compreensão científica que fundamenta toda a atividade de P\&D das firmas. Na científica básica, esses avanços têm impulsionado as taxas de melhoria tecnológica em uma ampla gama de setores e têm sido um requisito fundamental para a maioria do progresso tecnológico realizado desde o último século; ii) ao progresso tecnológico que tem origem em outras indústrias, ou seja, as oportunidades tecnológicas que surgem dos avanços tecnológicos no restante da economia; e iii) à existência de retornos positivos (feedbacks), pois em muitos setores industriais a pesquisa que é realizada no presente gera novos conhecimentos e aumenta as oportunidades tecnológicas futuras.

Essas fontes de oportunidade tecnológica que surgem nos setores industriais são moldadas e induzidas por um arcabouço institucional formado por organizações, convenções e normas sociais em geral. De um amplo conjunto de fatores institucionais existentes, o efeito da regulação é certamente o aspecto mais investigado pela literatura, principalmente quanto à eficácia de padrões ambientais, tecnológicos e baseados em desempenho, e nos instrumentos de mercado, tais como: impostos piguvianos ${ }^{9}$, subsídios, sistemas de depósito/restituição e licenças negociadas.

Esse debate é fortemente influenciado pela repercussão dos argumentos propostos por Porter (1991). O que ficou conhecido como Hipótese de Porter sugere que a regulação ambiental pode ter um efeito positivo sobre o desempenho de empresas nacionais em relação aos seus concorrentes estrangeiros, estimulando a inovação doméstica. Posteriormente, Porter e Van der Linde (1995) foram além ao afirmar que as

\footnotetext{
${ }^{9}$ Também conhecidos como taxas piguvianas são tributos aplicados a uma determinada atividade econômica que é passível de geração de externalidades negativas.
} 
regulações ambientais não apenas pressionam as firmas a inovar, mas também estimulam o seu crescimento e competitividade.

O argumento de Porter e Van der Linde (1995) é que o debate em termos da existência de um trade-off entre benefício social e custos privados desconsidera o novo paradigma de competitividade internacional baseado em inovação que emergiu nas décadas de 1980 e 1990. Nesse paradigma, a competitividade no nível industrial é resultado do alcance de uma produtividade superior, tanto em termos de baixos custos em relação aos rivais quanto da habilidade de ofertar produtos com valor superior. Desta forma, as evidências revelam que firmas alcançam competitividade não por operações em larga escala e com insumos baratos, mas com contínuo aprimoramento e inovação.

Ao avaliar os efeitos de políticas sobre a difusão tecnológica, Jaffe e Stavins (1995) contrariam a visão convencional e mostram que os subsídios de adoção possuem impacto superior à aplicação de taxas piguvianas. Em outro estudo sobre gastos ambientais e inovação em um painel de setores industriais, Jaffe e Palmer (1997) revelam que maiores gastos com conformidade ambiental levam a um aumento de investimento em P\&D.

De forma geral, os estudos empíricos mostram que as normas ambientais afetam a inovação das empresas, assim como maiores pressões regulatórias e normativas em questões ambientais influenciam positivamente a propensão das empresas a apostar na inovação ambiental (BERRONE et al., 2013; KESIDOU; DEMIREL, 2012). Contudo, segundo Brunnermeier e Cohen (2003), a expansão do monitoramento e da fiscalização da regulação existente não oferecem nenhum incentivo adicional para inovar.

Os resultados sobre a eficiência dos incentivos regulatórios não são um consenso na literatura, pois existe uma variação substancial nos resultados obtidos por instrumento e pelas políticas (VEUGELERS, 2012). Downing e White (1986) e Milliman e Príncipe (1989) argumentam que os incentivos a inovar são mais fortes em sistemas baseados no mercado, como taxas de emissão ou licenças, do que sob os regulamentos de comando e controle.

Nas abordagens baseadas em mercado, as justificativas para as intervenções estão baseadas na correção de externalidade e falhas de mercado, ainda que os diagnósticos variem na sua intensidade e urgência. Nordhaus (2008) afirma que essas intervenções devem ser graduais e limitadas a um período de tempo, e que a regulamentação ótima deve limitar o crescimento econômico apenas em uma pequena quantidade. Para Acemoglu et al. (2014), uma regulação ótima por um determinado período seria suficiente para redirecionar a mudança técnica. De qualquer forma, o sacrifício de parte do crescimento de longo prazo é inevitável. Já o diagnóstico de Stern (2008) é menos otimista e defende intervenções mais extensas e imediatas, ainda que possam implicar em significativos custos econômicos.

Como as políticas podem afetar o preço e a quantidade relativa do uso dessas tecnologias, Nordhaus (2008) afirma que a forma adequada de enviar um sinal correto para um conjunto amplo e diversificado de atores é a instituição de uma taxa de carbono que, para ter o efeito desejável, precisa ser global. O autor também avalia que o sistema de cap and trade ${ }^{10}$ não é capaz de enviar um sinal confiável, pois induz a uma forte flutuação nos preços de emissões e também está sujeito a um excessivo rent-seeking.

Para Foray, Mowery, Nelson (2012) e Mazzucato (2016), as mudanças climáticas devem ser tratadas pelas políticas de inovação como desafios socioeconômicos-tecnológicos (socioeconomic-technological

\footnotetext{
${ }^{10}$ Nesse sistema, o governo estabelece um comércio de emissões em que mecanismos de mercado são utilizados para controlar a poluição por meio do fornecimento de incentivos econômicos para alcançar reduções nas emissões de gases poluentes.
} 
challenges). Desta maneira, as estratégias tradicionais baseadas nas falhas de mercado devem ser supridas pela ação mais ativa na criação de mercado. São exemplos as políticas de inovação que ajudam a reduzir os custos nas fases de invenção e introdução no mercado por meio do apoio financeiro para projetos-piloto, e, na fase de difusão, na melhoria das características de desempenho das inovações. Já a política ambiental é responsável pela internalização dos custos externos impostos pela concorrência na fase de difusão ${ }^{11}$.

Existem divergências relevantes a respeito da extensão e da forma destas políticas para induzir a busca e a inovação em tecnologias mitigação e adaptação às mudanças climáticas. Para muitos autores, a dimensão dos problemas envolvidos e o pouco tempo disponível para implementação de suas soluções torna urgente a efetivação de grandes programas governamentais, com dimensão e escala análogos ao Manhattan Project (AMIDON, 2005; MICHAELSON, 1998; READ; LERMIT, 2005; SOMERVILLE, 2006) e ao Apollo Program (DUNN, 2002; JACOBSON, 2005). Mais recentemente, outro grupo de autores tem reivindicado um Green New Deal (DIPESO, 2009; FRIEDMAN, 2007; GROUP, 2008).

Do outro lado deste debate, Mowery, Nelson e Martin (2010) afirmam que os modelos baseados em grandes programas governamentais são inapropriados, pois emulam políticas usadas no passado para enfrentar desafios atuais que possuem características próprias. ${ }^{12}$ Os projetos Apolo e Manhattan foram programas governamentais muito particulares, elaborados, financiados e geridos por agências federais com objetivos específicos, e que tiveram o governo como o único cliente. Por outro lado, os autores consideram que as mudanças climáticas exigirão o envolvimento de atores heterogêneos, dispersos geograficamente e que precisarão empenhar expressivos volumes de recursos privados e públicos. Portanto, as tecnologias requeridas deverão atender à necessidade de diversas indústrias em vários níveis de complexidade, de novos motores a turbinas eólicas, do setor de transporte ao setor energético. Entretanto, as tecnologias representam apenas uma parte da solução do problema, mesmo que concordem com a necessidade de políticas tecnológicas governamentais. Para os autores, a natureza dos desafios é substancialmente diferente, ainda que os impactos previstos pelas mudanças climáticas suscitem questões técnicas e econômicas que podem inclusive ser mais complexas que uma aterrissagem lunar ou o desenvolvimento de bombas atômicas.

Mazzucato (2016) também afirma que as políticas atuais podem aprender com a abordagem orientada por missões (mission-oriented), principalmente quando é necessário que os setores público e privado trabalhem juntos no processo de busca e na criação de novas tecnologias e setores. Nessa abordagem, o lado público dessas parcerias não se limita a incentivar, facilitar, ou diminuir os riscos do setor privado. Mais do que isso, o risco do lado público é assumido por meio da escolha de uma determinada direção da busca tecnológica.

Entretanto, as propostas de políticas tecnológicas inspiradas em ênfase aos objetivos públicos dos anos 1950 e 1960 precisam considerar as diferenças fundamentais entre os projetos mission-oriented e novos projetos para enfrentamento de problemas ambientais.

O problema das abordagens que sustentam as políticas apenas em termos de correção de falhas e externalidades é a dificuldade de incorporar justificativas para a criação de mercados orientados para uma missão, como é o caso das mudanças climáticas. Da mesma forma, por não considerar o Estado como um condutor de investimentos e criador de mercado, as abordagens baseadas em mercado não fornecem uma orientação sobre o tipo e a estrutura das organizações do setor público que são necessárias para garantir e

\footnotetext{
${ }^{11}$ Independentemente da estrutura da intervenção adotada, é consenso que a ausência de punição pelos impactos nocivos ao meio ambiente, via mecanismos de mercados, tem como resultado uma distorção na concorrência entre as inovações ambientais e não ambientais.

${ }^{12}$ Em sentido figurado é como colocar um vinho novo em uma garrafa velha (putting new wine in old bottles).
} 
expandir a busca em tecnologias de alto risco. Por fim, a perspectiva limitada apenas à intervenção dificulta a avaliação dos investimentos e a distribuição dos riscos e benefícios entre o Estado e o setor privado (MAZZUCATO, 2016).

Para além da avaliação de políticas e regulações mais adequadas ao enfrentamento das mudanças climáticas, os estudos revelam um amplo conjunto de iniciativas existentes e já testadas em diversos países em todo mundo. Essas ações têm resultado na modificação dos incentivos para exploração dessas tecnologias. O exemplo principal é o efeito das políticas implementadas no contexto da assinatura do protocolo de Kyoto que afetam fortemente a busca tecnológica e a direção do progresso técnico em áreas de mitigação das mudanças climáticas (DECHEZLEPRÊTRE et al., 2011).

\section{Conclusões}

Este artigo tem como objetivo examinar o processo de mudanças climáticas globais como fator exógeno que modifica o grau e a direção do comportamento de busca tecnológica das firmas. Para tanto, analisa esse processo como um fator exógeno em três níveis: da firma, do mercado e do sistema. No primeiro nível, examina os fatores que podem estimular firmas a investir em processos de busca, a destacar o esgotamento tecnológico e a expansão para além de mercados nacionais. No segundo, analisa as condições específicas da sua formação e evolução que afetam dos mercados, a distribuição de incentivos e a existência de falhas e externalidades. Por fim, o terceiro nível avalia os incentivos para realização de atividades inovativas, a partir de diferentes condições de oportunidade tecnológica.

A revisão de literatura revela que enquanto estudos tradicionais enfatizaram o processo de busca local, a literatura subsequente procurou ampliar esse debate para um foco maior em como as firmas equilibram a busca local com a busca exploratória (não local). Essa abordagem teve como objetivo interpretar como firmas evitam a armadilha da busca local e equilibram a busca local com a busca exploratória. A mudança de ênfase parte do reconhecimento dos problemas da dependência da busca local.

A partir deste contexto, o artigo procura lidar com a ausência de estudos sobre fatores exógenos que modificam o nível e a direção do comportamento de busca das firmas. Ao mesmo tempo, também reconhece os desafios em identificar esses fatores, que precisam ser suficientemente abrangentes, ao mesmo tempo que independente de efeitos setoriais, tecnológicos ou locacionais. Percebe-se que os efeitos das mudanças climáticas sobre o comportamento das firmas constituem um contexto oportuno para investigação. A mitigação das emissões GEE é um desafio em escala global e exige a reestruturação de importantes ativos tecnológicos. Como consequência, as tecnologias de mitigação tendem a ser abrangentes, horizontais e pervasivas, e essas características exigem significativas modificações nos padrões de busca tecnológica.

Por fim, sem pretensão de esgotar o debate, o artigo constitui um esforço sistemático de interpretação de como as transformações no ambiente tecnológico, geradas pelas mudanças climáticas, atuam como um elemento exógeno capaz de alterar o grau e a direção do comportamento de busca tecnológica das firmas. Nesse sentido, também contribui para a literatura existente ao relacionar transformações em diferentes níveis, ao mesmo tempo em que extrapola os limites setoriais, geográficos e tecnológicos dos estudos existentes. 


\section{Referências}

ACEMOGLU, D.; AGHION, P.; HÉMOUS, D. The environment and directed technical change in a NorthSouth model. Oxford Review of Economic Policy, v. 30, n. 3, p. 513-530, 2014.

ADLER, P. S. et al. Perspectives on the Productivity Dilemma. Journal of Operations Management, v. 27, n. 2, p. 99-113, 2009.

AHUJA, G.; KATILA, R. Where do resources come from? The role of idiosyncratic situations. Strategic Management Journal, v. 25, n. 8-9, p. 887-907, 2004.

ALIC, J. A; MOWERY, D. C.; RUBIN, E. S. U.S. technology and innovation polices: Lessons for Climate Change. Arlington, VA: [s.n.].

AMIDON, J. M. America's Strategic Imperative, A “Manhattan Project"for Energy. Joint Forces Quarterly, v. 39, p. 68-77, 2005.

ARORA, A.; FOSFURI, A.; GAMBARDELLA, A. Markets for technology: the economics of innovation and corporate strategy. [s.1.] MIT, 2004.

BERNDT, E. R.; PINDYCK, R. S.; AZOULAY, P. Consumption externalities and Diffusion in Pharma markets: Antiulcer Drugs: NBER Working Paper. [s.l: s.n.].

BERRONE, P. et al. Necessity as the mother of "green" inventions: Institutional pressures and environmental innovations. Strategic Management Journal, v. 34, n. 8, p. 891-909, 2013.

BRUNNER, D. J. et al. Wellsprings of creation: How perturbation sustains exploration in mature organizations. Harvard Business School Working Paper No 09-011, p. 09-011, 2009.

BRUNNERMEIER, S. B.; COHEN, M. A. Determinants of environmental innovation in US manufacturing industries. Journal of Environmental Economics and Management, v. 45, n. 2, p. 278-293, 2003.

CANTWELL, J. A. Blurred Boundaries between Firms, and New Boundaries within (Large Multinational) Firms: The Impact of Decentralized Networks for Innovation. Seoul Journal of Economics, v. 26, n. 1, p. $1-32,2013$.

CANTWELL, J. A.; FAI, F. Firms as the source of innovation and growth: the evolution of technological competence. Journal of Evolutionary Economics, v. 9, p. 331-366, 1999.

COHEN, W. M.; LEVINTHAL, D. Absorptive capacity: a new perspective on learning and innovation. Administrative science quarterly, v. 35, n. 1, p. 128-152, 1990.

COHEN, W. M.; LEVINTHAL, D. A. Innovation and Learning : The Two Faces of R \& D. The Economic Journal, v. 99, n. 397, p. 569-596, 1989.

DECHEZLEPRETTRE, A. et al. Invention and transfer of climate change-mitigation technologies: A global analysis. Review of Environmental Economics and Policy, v. 5, n. 1, p. 109-130, 2011.

DIPESO, J. Sustainable engineering. Environmental Quality Management, v. 18, n. 3, p. 93-98, 2009. 
DOSI, G. Technological paradigms and technological trajectories. A suggested interpretation of the determinants and directions of technical change. Research Policy, v. 11, n. 3, p. 147-162, 1982.

DOSI, G. The Nature of the Innovative Process. In: DOSI, G. et al. (Eds.). . Technical Change and Economic Theory. London: Pinter Publishers, 1988. p. 221-238.

DOSI, G.; NELSON, R. R.; WINTER, S. G. Introduction: The Nature and Dynamics of Organizational Capabilities. In: The Nature and Dynamics of Organizational Capabilities. $1^{\text {a }}$ ed. Oxford: Oxford University Press, 2001. p. 1-22.

DOWNING, P. B.; WHITE, L. J. Innovation in pollution control. Journal of Environmental Economics and Management, v. 13, n. 1, p. 18-29, 1986.

DUNN, S. Hydrogen futures: toward a sustanable energy system. International Journal of Hydrogen Energy, v. 27, p. 235-264, 2002.

FLEMING, L. Recombinant Uncertainty in Technological Search. Management Science, v. 47, n. 1, p. $117-132,2001$.

FLEMING, L.; SORENSON, O. Science as a map in technological search. Strategic Management Journal, v. 25, n. 8-9, p. 909-928, 2004.

FORAY, D.; MOWERY, D. C.; NELSON, R. R. Public R\&D and social challenges: What lessons from mission R\&D programs? Research Policy, v. 41, n. 10, p. 1697-1702, 2012.

FOSFURI, A.; TRIBÓ, J. A. Exploring the antecedents of potential absorptive capacity and its impact on innovation performance. Omega, v. 36, n. 2, p. 173-187, 2008.

FREEMAN, C.; SOETE, L. The economics of industrial innovation. [s.1.] MIT Press, 1997.

FRIEDMAN, T. L. The Power of Green. The New York Times, 2007.

GAVETTI, G.; LEVINTHAL, D. Looking Forward and Looking Backward: Cognitive and Experiential Search. Administrative Science Quarterly, v. 45, n. 1, p. 113-137, 2000.

GROUP, G. N. D. A Green New Deal. London: New Economics Forum, 2008.

GUPTA, A. K. et al. The Interplay between Exploration and Exploitation Linked references are available on JSTOR for this article: THE INTERPLAY BETWEEN EXPLORATION AND EXPLOITATION. v. 49, n. 4, p. 693-706, 2006.

HE, Z.-L.; WONG, P.-K. Exploration vs. Exploitation: An Empirical Test of the Ambidexterity Hypothesis. Organization Science, v. 15, n. 4, p. 481-494, 2004.

JACOBSON, M. Z. Cleaning the Air and Improving Health with Hydrogen Fuel-Cell Vehicles. Science, v. 308, n. 5730, p. 1901-1905, 2005.

JAFFE, A. B. Technological Opportunity and Spillovers of R \& D: Evidence from Firms' Patents, Profits, and Market Value. The American Economic Review, v. 76, n. 5, p. 984-1001, 1986. 
JAFFE, A. B.; NEWELL, R. G.; STAVINS, R. N. A tale of two market failures: Technology and environmental policy. Ecological Economics, v. 54, n. 2-3, p. 164-174, 2005.

JAFFE, A. B.; PALMER, K. Environmental regulation and innovation: a panel data study. Review of economics and statistics, v. 79, n. 4, p. 610-619, 1997.

JAFFE, A. B.; STAVINS, R. N. Dynamic Incentives of Environmental Regulations: The Effects of Alternative Policy Instruments on Technology Diffusion. Journal of Environmental Economics and Management, v. 29, n. 3, p. S43-S63, 1995.

JANSEN, J. J. P.; VAN DEN BOSCH, F. A. J.; VOLBERDA, H. W. Managing potential and realized absorptive capacity: How do organizational antecedents matter? Academy of Management Journal, v. 48, n. 6, p. 999-1015, 2005.

JONES, C. I. Sources of U.S. Economic Growth in a World of Ideas. The American Economic Review, v. 92, n. 1, p. 220-239, 2002.

JONES, C. I.; WILLIAMS, J. C. Measuring the Social Return to R\&amp;D. The Quarterly Journal of Economics, v. 113, n. 4, p. 1119-1135, 1 nov. 1998.

KATILA, R.; AHUJA, G. Something Old, Something New: A Longitudinal Study of Search Behavior and New Product Introduction. Academy of Management, v. 45, n. 6, p. 1183-1194, 2002.

KATZ, R.; ALLEN, T. Investigating the Not Invented Here syndrom e: a look at the perform ance, tenure and com $m$ unification patterns of 50 R\& D project groups. R\&D Management, v. 12, n. 1956, p. 7-19, 1982.

KESIDOU, E.; DEMIREL, P. On the drivers of eco-innovations: Empirical evidence from the UK. Research Policy, v. 41, n. 5, p. 862-870, 2012.

KLEVORICK, A. K. et al. Policy in Technological Opportunities. Research in Economics, v. 24, p. 185205, 1995.

KNUDSEN, T.; LEVINTHAL, D. A. Two Faces of Search: Alternative Generation and Alternative Evaluation. Organization Science, v. 18, n. 1, p. 39-54, 2007.

LANE, P. J.; KOKA, B. R.; PATHAK, S. The reification of absorptive capacity: A critical review and rejuvenation of the construct. Academy of Management Review, v. 31, n. 4, p. 833-863, 2006.

LAURSEN, K. Keep searching and you'll find: What do we know about variety creation through firms' search activities for innovation? Industrial and Corporate Change, v. 21, n. 5, p. 1181-1220, 2012.

LAURSEN, K.; SALTER, A. Open for innovation: The role of openness in explaining innovation performance among U.K. manufacturing firms. Strategic Management Journal, v. 27, n. 2, p. 131-150, 2006.

LAVIE, D.; STETTNER, U.; TUSHMAN, M. L. Exploration and Exploitation Within and Across Organizations. Academy of Management Annals, v. 4, n. 1, p. 109-155, 2010.

LEVINTHAL, D. A.; MARCH, J. G. the Myopia of Learning. Strategic Management Journal, v. 14, p. 
95-112, 1993.

MALERBA, F.; ORSENIGO, L. Technological regimes and firm behaviour. Industrial and Corporate Change, v. 19, n. 1, p. 45-71, 1993.

MARCH, J. G. Exploration and Exploitation in Organizational Learning. Organization Science, v. 2, n. 1, p. 71-87, fev. 1991.

MAS-COLELL, A.; WHINSTON, M. D.; GREEN, J. R. Microeconomic theory. New York, NY: Oxford University Press, 1995.

MAZZUCATO, M. From market fixing to market-creating: a new framework for innovation policy. Industry and Innovation, v. 23, n. 2, p. 140-156, 2016.

MICHAELSON, J. Geoengineering: A Climate Change Manhattan Project. Stanford Environmental Law Review, v. 17, p. 73, 1998.

MILLIMAN, S. R.; PRINCE, R. Firm incentives to promote technological change in pollution control. Journal of Environmental Economics and Management, v. 17, n. 3, p. 247-265, 1989.

MOWERY, D. C.; NELSON, R. R.; MARTIN, B. R. Technology policy and global warming: Why new policy models are needed (or why putting new wine in old bottles won't work). Research Policy, v. 39, n. 8, p. 1011-1023, 2010.

NELSON, R. R. Why Do Firms Differ, and How Does it Matter? Strategic Management Journal, v. 12, n. Special Issue: Fundamental Research Issues, p. 61-74, 1991.

NELSON, R. R.; WINTER, S. G. An evolutionary theory of economic change. Cambridge: Belknap Press of Harvard University Press, 1982.

NORDHAUS, W. D. A question of balance : weighing the options on global warming policies. New Haven, EUA: Yale University Press, 2008.

PATEL, P. R.; PAVITT, K. The technological competencies of the world's largest firms: Complex and path-dependent, but not much variety. Research Policy, v. 26, n. 2, p. 141-156, 1997.

PAVITT, K. Technologies, Products and Organization in the Innovating Firm: What Adam Smith Tells Us and Joseph Schumpeter Doesn’t. Industrial and Corporate Change, p. 433-452, 1998.

POPP, D.; HAFNER, T.; JOHNSTONE, N. Environmental policy vs. public pressure: Innovation and diffusion of alternative bleaching technologies in the pulp industry. Research Policy, v. 40, n. 9, p. 1253$1268,2011$.

PORTER, M. E. America's green strategy. Scientific American, v. 264, n. 4, p. 168, 1991.

PORTER, M. E.; VAN DER LINDE, C. Toward a New Conception of the Environmend-Competetiveness Relationship. The Journal of Economic Perspectives, v. 9, n. 4, p. 97-118, 1995.

READ, P.; LERMIT, J. Bio-energy with carbon storage (BECS): A sequential decision approach to the threat of abrupt climate change. Energy, v. 30, n. 14, p. 2654-2671, 2005. 
RENNINGS, K. Redefining innovation-eco-innovation research and the contribution from ecological economics. Ecological economics, v. 32, n. 2, p. 319-332, 2000.

ROSENKOPF, L.; ALMEIDA, P. Overcoming Local Search Through Alliances and Mobility. Management Science, v. 49, n. 6, p. 751-766, 2003.

ROSENKOPF, L.; NERKAR, A. Beyond local search: Boundary-spanning, exploration, and impact in the optical disk industry. Strategic Management Journal, v. 22, n. 4, p. 287-306, 2001.

ROTHAERMEL, F. T.; ALEXANDRE, M. T. Ambidexterity in Technology Sourcing: The Moderating Role of Absorptive Capacity. Organization Science, v. 20, n. 4, p. 759-780, 2009.

SIMON, H. A. Models of Bounded Rationality, Volume 3. Cambridge, MA, and London, UK: MIT Press, 1997.

SOMERVILLE, C. The Billion-Ton Biofuels Vision. Science, v. 312, n. 5778, p. 1277-1277, 2006.

STERN, N. The economics of climate change. American Economic Review, v. 98, n. 2, p. 1-37, 2008.

TRIPSAS, M.; GAVETTI, G. Capabilities,Cognition, and Inertia: Evidence from digital imaging. Strategic Management Journal, v. 21, n. 10, p. 1147-1161, 2000.

TUSHMAN, M. L.; O'REILLY, C. A. The Ambidextrous Organizations: Managing Evolutionary and Revolutionary Change. California Management Review, v. 38, n. 4, p. 8-30, 1996.

VERMEULEN, F.; BARKEMA, H. Learning through Acquisitions. The Academy of Management Journal, v. 44, n. 3, p. 457-476, 2001.

VEUGELERS, R. Which policy instruments to induce clean innovating? Research Policy, v. 41, n. 10, p. 1770-1778, 2012.

WINTER, S. G. Schumpeterian competition in alternative technological regimes. Journal of Economic Behavior and Organization, v. 5, n. 3-4, p. 287-320, 1984.

ZAHRA, S. A.; GEORGE, G. Absorptive Capacity : a Review, and Extension. Academy of Management Review, v. 27, n. 2, p. 185-203, 2002. 